\title{
Off-pump coronary artery bypass sacrifices graft patency: Meta-analysis of randomized trials
}

\author{
Hisato Takagi, MD, PhD, Toshiyuki Tanabashi, MD, Norikazu Kawai, MD, Takayoshi Kato, MD, \\ and Takuya Umemoto, MD, PhD, Shizuoka, Japan
}

See related editorial on page 1.

$\mathrm{T}$ he most recent meta-analysis ${ }^{1}$ of 37 randomized trials of off-pump coronary artery bypass (OPCAB) versus conventional coronary artery bypass grafting $(\mathrm{CABG})$ demonstrated that mortality, stroke, myocardial infarction, and renal failure were not reduced in $\mathrm{OPCAB}$; however, selected short-term and midterm clinical and resource outcomes were improved compared with CABG. The previous cumulative analysis (by Parolari and associates ${ }^{2}$ ) of 5 prospective randomized studies (by Nathoe, ${ }^{3}$ Khan, ${ }^{4}$ Puskas, ${ }^{5}$ Widimsky, ${ }^{6}$ Lingaas, ${ }^{7}$ and their associates) then available in the literature, however, documented a reduction in postoperative patency of bypass grafts performed during OPCAB procedures. Since the meta-analysis by Parolari and associates ${ }^{2}$ was conducted, Lingaas and colleagues ${ }^{8}$ have updated the 3 -month patency, ${ }^{7}$ and Kobayashi and coworkers ${ }^{9}$ and Al-Ruzzeh and associate ${ }^{10}$ have reported results of other randomized controlled trials. In these trials, ${ }^{8-10} \mathrm{OPCAB}$ provided the same angiographic graft patency as CABG, despite the conclusion of the meta-analysis by Parolari and colleagues. ${ }^{2}$ To reassess differences in graft patency between OPCAB and CABG, we performed a meta-analysis of currently available randomized controlled trials of $\mathrm{OPCAB}$ versus $\mathrm{CABG}$.

\section{Materials and Methods}

All prospective randomized controlled trials that compared patency at least 3 months after OPCAB and CABG were identified using a 2-level search strategy. First, a public domain database (MEDLINE) was searched with a Web-based search engine (PubMed). Second, relevant studies were identified through a manual search of secondary sources including references of initially identified articles. The MEDLINE database was searched from January 1966 to June 2006. Keywords included "off-pump," "off pump," "opcab," "patency," and "randomized controlled trial." Studies considered for inclusion met the following criteria: The design was a

\footnotetext{
From the Department of Cardiovascular Surgery, Shizuoka Medical Center Shizuoka, Japan.

Received for publication Aug 10, 2006; accepted for publication Aug 25, 2006.

Address for reprints: Hisato Takagi, MD, PhD, Department of Cardiovascular Surgery, Shizuoka Medical Center, 762-1 Nagasawa, Shimizu-cho, Sunto-gun, Shizuoka 411-8611, Japan (E-mail: kfgth973@ybb.ne.jp).

J Thorac Cardiovasc Surg 2007;133:e2-3

$0022-5223 / \$ 32.00$

Copyright $\odot 2007$ by The American Association for Thoracic Surgery doi:10.1016/j.jtcvs.2006.08.062
}

prospective randomized controlled clinical trial; patients were randomly assigned to OPCAB versus $\mathrm{CABG}$; and main outcomes included at least 3-month graft patency evaluated by angiography. All qualifying studies were assessed for adequate blinding of randomization, completeness of follow-up, and objectivity of the outcome assessment. Data regarding arterial graft and venous graft (excluding radial artery graft as available) patency were abstracted from each individual study. For each study, data regarding patency in both the $\mathrm{OPCAB}$ and $\mathrm{CABG}$ groups were used to generate risk ratios (RRs) $(<1$, favors OPCAB; $>1$, favors $\mathrm{CABG}$ ) and risk differences (RDs) $(<0$, favors $\mathrm{OPCAB}$; $>0$, favors $\mathrm{CABG})$ for graft "occlusion" and 95\% confidence intervals (CIs). Study-specific estimates were combined with a random-effect model. Between-study heterogeneity was analyzed by standard $\chi^{2}$ tests. Sensitivity analyses were performed to assess the contribution of each study to the pooled estimate by excluding individual trials one at a time and recalculating the pooled $\mathrm{RR}$ and RD estimates for the remaining studies. Publication bias was assessed graphically with a funnel plot and mathematically with an adjusted rank-correlation test.

\section{Results}

Our search identified 6 prospective randomized controlled clinical trials $^{3-6,8,10}$ (Table 1). We excluded the trial by Kobayashi and associates $^{9}$ because these workers merely examined early (within 3 weeks after the operation) graft patency. Allocation concealment and blinding were not possible in these trials given that the intervention was surgical; however, all trials used a blinded committee for adjudication of events. Careful accounting for dropouts and crossovers was provided in all cases, and all but one analysis ${ }^{5}$ were conducted by intention-to-treat. All of the 6 individual trials demonstrated a statistically nonsignificant benefit of CABG over OPCAB for overall graft patency. Pooled analysis demonstrated a statistically significant $27 \%$ increase in overall graft "occlusion" with OPCAB relative to CABG (RR, 1.27; 95\% CI, 1.03-1.56; $P=.0234)(\mathrm{RD}, 3.0 \%$; 95\% CI, $0.6 \%-5.4 \% ; P=.0129)$. There was neither trial heterogeneity of results nor evidence of significant publication bias. Exclusion of any single trial from the analysis did not substantively alter the overall result of our analysis. Subanalyses demonstrated a statistically nonsignificant benefit of CABG over OPCAB for arterial graft patency (RR, 1.17; 95\% CI, 0.62-2.24; $P=.6259)(\mathrm{RD}, 2.0 \%$; 95\% CI, $-1.2 \%-5.2 \% ; P=.2195)$ and a statistically significant $28 \%$ increase in venous graft "occlusion" with OPCAB relative to CABG (RR, $1.28 ; 95 \%$ CI, 1.06-1.54; $P=.0094)(\mathrm{RD}, 4.0 \%$; 95\% CI, $0.2 \%$ $7.8 \% ; P=.0396)$.

\section{Discussion}

The present meta-analysis demonstrated a significant increase in overall graft "occlusion," especially in venous graft "occlusion," with OPCAB relative to $\mathrm{CABG}$. On the one hand, OPCAB de- 
TABLE 1. Characteristics of trials and meta-analysis of graft patency

\begin{tabular}{|c|c|c|c|c|c|c|c|}
\hline & Al-Ruzzeh ${ }^{10}$ & Lingaas $^{8}$ & Widimsky $^{6}$ & Puskas $^{5}$ & Khan $^{4}$ & Nathoe $^{3}$ & Combined \\
\hline No. of patients & 168 & 120 & 400 & 197 & 103 & 110 & 1098 \\
\hline Timing of CAG, mo & 3 & 12 & 12 & 12 & 3 & 12 & 9 \\
\hline Patients undergoing CAG, \% & 90 & 91 & 64 & 78 & 80 & 64 & 75 \\
\hline Patients features & $\begin{array}{l}\text { Isolated, elective } \\
\text { CAS }\end{array}$ & Elective CAS & $\begin{array}{l}\text { Isolated, elective } \\
\text { CAS }\end{array}$ & $\begin{array}{l}\text { Isolated, primary, } \\
\text { elective CAS }\end{array}$ & $\begin{array}{l}\text { Isolated, primary, } \\
\text { CAS }\end{array}$ & $\begin{array}{l}\text { Isolated, } \\
\text { primary, } \\
\text { elective CAS }\end{array}$ & \\
\hline Intention-to-treat & No crossovers & Yes & No & Yes & Yes & Yes & \\
\hline Events committee & Blinded & NR & NR & Blinded & Blinded & Blinded & \\
\hline \multicolumn{8}{|l|}{ Arterial graft patency } \\
\hline $\mathrm{OPCAB}, \mathrm{n} / \mathrm{N}^{*}(\%)$ & $\begin{array}{l}111 / 113(98) \dagger \\
(50 / 55[91]) \S\end{array}$ & $48 / 51(94) \dagger$ & 98/107 (91)† & $95 / 101(94) \ddagger$ & $\begin{array}{l}46 / 50(92) \dagger \\
(26 / 34[76]) \S\end{array}$ & NR & $398 / 422(94)$ \\
\hline CABG, n/N* $(\%)$ & $\begin{array}{l}111 / 114(97) \dagger \\
(44 / 50[88]) \S\end{array}$ & $54 / 56(96) \dagger$ & $100 / 110(91) \dagger$ & 102/104 (98)‡ & $\begin{array}{l}47 / 47(100) \dagger \\
(22 / 22[100]) \S\end{array}$ & NR & $414 / 431(96)$ \\
\hline $\mathrm{RR} \|(95 \% \mathrm{Cl})$ & $0.67(0.11-3.95)$ & $1.65(0.29-9.46)$ & $0.93(0.39-2.19)$ & $3.09(0.64-14.95)$ & - & - & $1.17(0.62-2.24)$ \\
\hline RDף $(95 \% \mathrm{CI}), \%$ & $-0.9(-4.7-3.0)$ & $2.3(-5.8-10.4)$ & $-0.7(-8.2-6.8)$ & $4.0(-1.3-9.3)$ & $8.0(0.5-15.5)$ & - & $2.0(-1.2-5.2)$ \\
\hline \multicolumn{8}{|l|}{ Venous graft patency } \\
\hline OPCAB, n/N* $(\%)$ & $50 / 61(82)$ & $67 / 84(80)$ & $86 / 176(49)$ & $140 / 150$ (93) & $40 / 44(91)$ & NR & $383 / 515$ (74) \\
\hline $\mathrm{CABG}, \mathrm{n} / \mathrm{N}^{*}(\%)$ & $60 / 68(88)$ & $84 / 97(87)$ & $145 / 246(59)$ & $147 / 156(94)$ & $56 / 59$ (95) & NR & $492 / 626(79)$ \\
\hline $\mathrm{RR} \|(95 \% \mathrm{Cl})$ & $1.53(0.66-3.56)$ & $1.51(0.78-2.92)$ & $1.25(1.01-1.53)$ & $1.16(0.48-2.76)$ & $1.79(0.42-7.58)$ & - & $1.28(1.06-1.54)$ \\
\hline RDI (95\% CI), \% & $6.3(-6.0-18.6)$ & $6.8(-4.1-17.8)$ & $10.1(0.5-19.7)$ & $0.9(-4.5-6.3)$ & $4.0(-6.2-14.2)$ & - & $4.0(0.2-7.8)$ \\
\hline \multicolumn{8}{|l|}{ Overall graft patency } \\
\hline OPCAB, n/N* $(\%)$ & 161/174 (93)\# & $115 / 135(85)$ & $197 / 283(70)$ & $235 / 251(94) \ddagger$ & 86/94 (91)\# & $63 / 69(91)$ & $857 / 1006$ (85) \\
\hline CABG, n/N* (\%) & 171/182 (94)\# & $138 / 153(90)$ & $264 / 356(74)$ & $249 / 260(96) \ddagger$ & 103/106 (97)\# & $83 / 89$ (93) & $1008 / 1146$ (88) \\
\hline $\mathrm{RR} \|(95 \% \mathrm{Cl})$ & $1.24(0.57-2.68)$ & $1.51(0.81-2.83)$ & $1.18(0.92-1.51)$ & $1.51(0.71-3.18)$ & $3.01(0.82-11.01)$ & $1.29(0.43-3.83)$ & $1.27(1.03-1.56)$ \\
\hline RDI (95\% CI), \% & $1.4(-3.8-6.6)$ & $5.0(-2.6-12.6)$ & $4.6(-2.5-11.6)$ & $2.1(-1.7-6.0)$ & $5.7(-0.8-12.1)$ & $2.0(-6.5-10.4)$ & $3.0(0.6-5.4)$ \\
\hline
\end{tabular}

CABG, Conventional coronary artery bypass grafting; CAG, coronary angiography; CAS, coronary artery surgery; Cl, confidence interval; NR, not reported; OPCAB, off-pump coronary artery bypass; $\mathrm{RD}$, risk difference; $R R$, risk ratio. $*$ No. of patent grafts/Total No. of grafts. $\dagger$ Patency of internal thoracic artery grafts. $\ddagger$ Patency including radial artery grafts. §Patency of radial artery grafts. "Risk ratio for graft "occlusion" $(<1$, favors OPCAB; $>1$, favors CABG). IRisk difference for graft "occlusion" $(<0$, favors $0 P C A B$; $>0$, favors $\mathrm{CABG})$. \#Patency excluding radial artery grafts.

creases arterial fibrillation, transfusion, inotrope requirements, respiratory infections, ventilation time, intensive care unit stay, and hospital stay. ${ }^{1}$ At the expense of graft patency, dare we perform OPCAB rather than CABG to merely improve these selected clinical and resource outcomes?

\section{References}

1. Cheng DC, Bainbridge D, Martin JE, Novick RJ, Evidence-Based Perioperative Clinical Outcomes Research Group. Does off-pump coronary artery bypass reduce mortality, morbidity, and resource utilization when compared with conventional coronary artery bypass? A meta-analysis of randomized trials. Anesthesiology. 2005;102:188-203.

2. Parolari A, Alamanni F, Polvani G, Agrifoglio M, Chen YB, Kassem S, et al. Meta-analysis of randomized trials comparing off-pump with on-pump coronary artery bypass graft patency. Ann Thorac Surg. 2005;80:2121-5.

3. Nathoe HM, van Dijk D, Jansen EW, Suyker WJ, Diephuis JC, van Boven WJ, et al. A comparison of on-pump and off-pump coronary bypass surgery in low-risk patients. $N$ Engl J Med. 2003;348:394-402.

4. Khan NE, De Souza A, Mister R, Flather M, Clague J, Davies S, et al. A randomized comparison of off-pump and on-pump multivessel coronary-artery bypass surgery. $N$ Engl J Med. 2004;350:21-8.
5. Puskas JD, Williams WH, Mahoney EM, Huber PR, Block PC, Duke PG, et al. Off-pump vs conventional coronary artery bypass grafting: early and 1-year graft patency, cost, and quality-of-life outcomes-a randomized trial. JAMA. 2004;291:1841-9.

6. Widimsky P, Straka Z, Stros P, Jirasek K, Dvorak J, Votava J, et al. One-year coronary bypass graft patency: a randomized comparison between off-pump and on-pump surgery-angiographic results of the PRAGUE-4 trial. Circulation. 2004;110:3418-23.

7. Lingaas PS, Hol PK, Lundblad R, Rein KA, Tonnesen TI, Svennevig JL, et al. Clinical and angiographic outcome of coronary surgery with and without cardiopulmonary bypass: a prospective randomized trial. Heart Surg Forum. 2004;7:37-41.

8. Lingaas PS, Hol PK, Lundblad R, Rein KA, Mathisen L, Smith H-J, et al. Clinical and radiologic outcome of off-pump coronary surgery at 12 months follow-up: a prospective randomized trial. Ann Thorac Surg. 2006;81:2089-96.

9. Kobayashi J, Tashiro T, Ochi M, Yaku H, Watanabe G, Satoh T, et al. Early outcome of a randomized comparison of off-pump and onpump multiple arterial coronary revascularization. Circulation. 2005;112(9 Suppl):I338-43.

10. Al-Ruzzeh S, George S, Bustami M, Wray J, Ilsley C, Athanasiou T, et al. Effect of off-pump coronary artery bypass surgery on clinical, angiographic, neurocognitive, and quality of life outcomes: randomised controlled trial. BMJ. 2006;332:1365. 